Gdańsk 2018, Nr. 38

\author{
Andriej Kotin \\ Uniwersytet Zielonogórski / Universität Zielona Góra \\ https://doi.org/10.26881/sgg.2018.38.05
}

\title{
Der Sprechende und das Unaussprechliche - Literarische Kunst als „Kampf mit der Sprache“
}

Der vorliegende Beitrag befasst sich mit dem Problem der Verhältnisse zwischen dem Schreibenden und der Schrift, dem Sprechenden und der Sprache, wobei unter der Letzteren ein unbegrenztes (Selbst) Ausdrucks- und Benennungspotenzial verstanden wird. Die wichtigsten Themen, die in diesem Kontext diskutiert werden - Literatur und andere Kunstformen, die Dialektik der Autor- und Erzählerkonzepte, die Idee des Unaussprechlichen usw. - kreisen um die Kernfrage: Ist es etwa der Autor, der die Sprache benutzt, um bestimmte Gedanken und Gefühle auszudrücken, oder wird er vielleicht selbst benutzt, ja überhaupt kreiert von einem ihm übergeordneten Phänomen, das man ,Sprache' nennt?

Schlüsselwörter: Sprache, Autor, Erzähler, das Unaussprechliche

The Speaker and the Unspeakable - The Art of Literature as a "Struggle with Language". The article focuses on the problem of relations between a writer and a language: between the one who speaks and speech itself, understood as a vast medium for virtually infinite (self-) expression. The most important topics to be discussed in this context include those of literature and other art forms, that of the dialectics of the concepts of 'the author' and 'the narrator' and that of the notion of the 'Ineffable'. These are considered in the light of one fundamental question namely whether the author uses language to express his or her own thoughts and feelings or, perhaps, is the author merely an agent or product of some greater and more widely unappreciated phenomenon called 'Language'?

Keywords: language, author, narrator, the unspeakable

Hermann Karlovich, der deutsch-russische Ich-Erzähler und zugleich die Hauptfigur von Vladimir Nabokovs Roman „Die Verzweiflung“, kommt auf die Idee, seinen vermeintlichen Doppelgänger - den einfälttigen Landstreicher Felix - umzubringen und damit den eigenen Tod vorzutäuschen. Nach Hermanns Plan sollte seine Ehefrau die Lebensversicherungsprämie bekommen, und danach könnten die Beiden andernorts ein neues, sorglos-luxuriöses Leben beginnen. Schließlich stellt sich heraus, dass die Ähnlichkeit zwischen Hermann und Felix lediglich in seiner Phantasie bestand, sodass kein Mensch auch nur auf die Idee kommt, den Ermordeten mit dem Täter zu verwechseln. Hermann wird also verhaftet und erzählt seine Geschichte, indem er sie als einen tragischen Versuch darstellt, ein perfektes Verbrechen zu vollbringen. Die Schuld dafür, dass sein geniales Kunstwerk letztendlich scheitert, trägt - so Hermann - die stumpfe Blindheit der Durchschnittsmenschen, welche nicht imstande sind, das zu bemerken, was nur einem wahren Künstler offenbart werden kann. In der Wirklichkeit ist aber die Hauptfigur des Romans natürlich kein wahrer Künstler, sondern 
gerade dessen Gegenpol und ein äußerst unzuverlässiger Erzähler, dessen zahlreiche Lügen und Fehltritte erst im Nachhinein ans Licht treten. Es gibt aber im Text eine Stelle, die nicht nur oft zitiert, sondern auch als besonders kennzeichnend für Nabokovs Kunstverständnis betrachtet wird. In dieser Szene beschreibt Hermann sein erstes Treffen mit dem Pseudo-Doppelgänger und macht nebenbei einige interessante Bemerkungen zum allgemeinen Wesen des Schrifttums:

[...] ich fürchte, dass Wörter, aufgrund ihrer besonderen Natur, allein nicht imstande sind, eine derartige Ähnlichkeit anschaulich zu vermitteln: Die beiden Gesichter müssten Seite an Seite abgebildet werden, in echten Farben, nicht in Worten; dann und nur dann würde der Zuschauer sehen, worum es mir geht. Es ist der Lieblingstraum eines Schriftstellers, den Leser in einen Zuschauer zu verwandeln; aber wird dies je erreicht? [...] im gegenwärtigen Augenblick brauche ich nicht literarische Methoden, sondern die ganz gewöhnliche, grobschlächtige Deutlichkeit der Malerei. ${ }^{1}$

Die Frage, ob und inwiefern es berechtigt wäre, diese Überlegungen auf Nabokov und sein Schreibkonzept zu übertragen, ist im Kontext des vorliegenden Artikels eher unwesentlich und wird daher nicht weiter diskutiert. Die Gedanken, die im oben zitierten Fragment zum Ausdruck kommen, sind allerdings als solche sehr bemerkenswert und aufschlussreich. Es wird hier nämlich postuliert, das Wort bzw. die Sprache, seien „aufgrund ihrer besonderen Natur " dazu unfähig, gewisse Sachverhalte bzw. Schattierungen wiederzugeben. Konsequenterweise stellt sich die Frage: Worin besteht nun diese ,besondere Natur' sprachlicher bzw. schriftlicher Äußerungen? Oder: Ist die erwähnte Nicht-Präzision ein ursprünglicher Bestand- und zugleich Nachteil der Sprache, da das unvermeidbare Scheitern eigentlicher Vermittlung in jeder (Wort)Benennung ontologisch impliziert wird? Hermann beantwortet diese Frage mit einem eindeutigen ,Ja', ohne auf kommentierende Einzelheiten einzugehen. Hinterfragt man aber die Gründe jenes Sprachskeptizismus bzw. Sprachpessimismus, der bereits in der englischen Philosophie des 17. Jh. entsteht ${ }^{2}$ und spätestens seit Heinrich von Kleist zum stets wiederkehrenden literarischen Motiv wird, so können die Ergebnisse dieser Reflexion einige beachtenswerte Parallelen aufweisen.

\section{Der „Kampf mit der Sprache“3}

Immanuel Kants erste Vorrede zu seiner „Kritik der reinen Vernunft“ beginnt mit einer markanten Feststellung, deren Geltungsbereich weit über das besprochene Problem hinausgeht:

Die menschliche Vernunft hat das besondere Schicksal in einer Gattung ihrer Erkenntnisse: daß sie durch Fragen belästigt wird, die sie nicht abweisen kann, denn sie sind ihr durch die Natur der Vernunft

Vladimir Nabokov, Die Verzweiflung, Reinbek bei Hamburg 2001, S. 21.

Vgl. Elisabeth Leiss, Sprachphilosophie, Berlin 2009, S. 83.

3 Dieser Begriff wird in einem äußerst interessanten philosophischen Kontext im Hauptwerk von Alexander Piatigorsky (1929-2009), einem hervorragenden russischen Philosophen, verwendet. Siehe dazu: Александр Пятигорский (Aleksandr Piatigorski), Мышление и наблюдение. Четыре кекции по обсервационной фимософии [Myschlenie i nabljudenie. Tschetyre lekcii po obserwacionnoj filosofii] (Denken und Beobachten. Vier Vorträge über die Observationsphilosophie), Moskau 2016, S. 22. 
selbst aufgegeben, die sie aber auch nicht beantworten kann, denn sie übersteigen alles Vermögen der menschlichen Vernunft. ${ }^{4}$

Kant gilt zwar nicht als Sprachphilosoph, denn die Sprache wird in seinem Denksystem, so Elisabeth Leiss, lediglich als „Mitarbeiterin der Vernunft im Außendienst“ “ betrachtet und somit zur bloßen Gedankenvermittlerin reduziert. Nichtsdestoweniger gibt Leiss der Behauptung von Josef Simon Recht, „kein Philosoph der Neuzeit habe eine größere Wirkung auf die Sprachphilosophie gehabt als Kant ${ }^{\text {“6 }}$. Machen wir nun ein kleines Umtauschexperiment und ersetzen wir ,Vernunft' durch ,Sprache', um den neuen Sinngehalt, der sich daraus ergibt, auf dessen semantische Legitimität hin zu prüfen. Auf epistemologische sowie onomastische Sprachfunktionen übertragen, hieße es also:

Die menschliche Sprache hat das besondere Schicksal in einer Gattung ihrer Erkenntnisse: dass sie sich zur Formulierung von Fragen eignet, die man nicht abweisen kann, denn sie sind dem Sprachverwender durch die Natur der Sprache selbst aufgegeben. Jene Fragen können aber mithilfe der Sprache nicht beantwortet werden, denn die übersteigen alles Vermögen der menschlichen Sprache.

Davon hinausgehend, könnte man der menschlichen Sprache ein sowohl autoreferentielles als auch selbsttranszendierendes Potenzial zuschreiben. Mehr noch: Dieses Potenzial wäre nicht etwa arbiträr zu verstehen (im Sinne sprachskeptischer Philosophen), sondern als eine natürliche ,angeborene' Eigenschaft der Sprache (ein obligatorischer Teil ihrer ursprünglichen ,Natur'). Eine solche Sprachauffassung erinnert einigermaßen an Friedrich Nietzsches berühmtes Postulat, der Mensch sei etwas, was überwunden werden soll.7 Die Sprache tendiere also danach, sich selbst zu überbrücken bzw. zu transzendieren. Mit anderen Worten: Das Streben, die Sprache niederzukämpfen, lässt sich nicht (oder wenigstens nicht nur) aus individueller menschlicher Willkür ableiten, sondern es entspringt vielmehr der transpersonalen (Ur-)Natur der Sprache. Letztere wird in diesem Zusammenhang als eine Art ,Ding an sich - nicht also pragmatisch/intentionell, sondern ontologisch/existentiell - verstanden. Es gibt jedoch eine sehr spezifische, obwohl allgemein verbreitete Ausnahmesphäre menschlicher Sprachbemächtigung, wo das Existentielle mit dem Intentionellen derartig dezent und zwanglos verschmilzt, dass der Unterschied zwischen ihnen beinahe unbemerkbar bleibt. Diese Sphäre nennt man Literatur.

Da ein narrativer Text in erster Linie, d.h. auf der rein (erzähl)technischen Ebene, gerade mit der Sprache arbeitet, bezieht sich die oben formulierte Kant-Paraphrase logischerweise auf jedes literarische Werk, das sich durch ein gewisses Stil- bzw. Schreibniveau auszeichnet (obschon auch künstlerisch anspruchslose Texte über die Basisdialektik von Wort und Bedeutung kaum hinweg kommen). Je subjektiver, je ,irregulärer' ein auszudrückender Gedanke oder eine im Roman enthaltene Idee sind, desto brennender wird das Problem von deren sprachlicher Vermittlung. Indem Nabokovs Protagonist (oder eher Antagonist) sich über die ,besondere Natur ' der Wörter

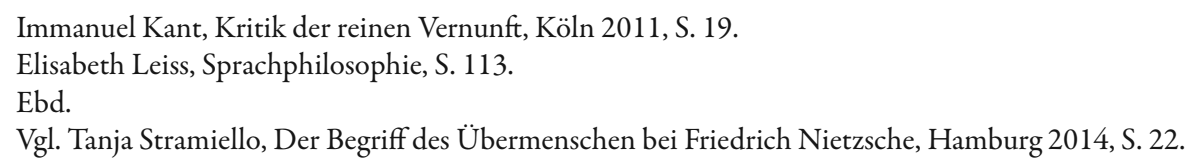


beschwert, klagt er eben diejenigen, natürlichen ' Grenzen menschlicher Sprache an, die mit den Grenzen menschlicher Vernunft in Kants Philosophie zwar keineswegs identisch, aber teilweise vergleichbar sind. Genauer gesagt geht es Hermann Karlovich darum, dass ein Wort niemals so anschaulich wirken kann wie ein Bild. In seinem Wunsch, den Leser in einen Zuschauer zu verwandeln, ist die übergeordnete Position der Malerei gegenüber der literarischen Kunst einbegriffen, denn letztere sei nicht imstande, die (imaginäre) Ähnlichkeit zwischen Hermann und Felix wiederzugeben. Die Sprachkritik des Ich-Erzählers klingt wie ein polemischer Gegensatz zu Ludwig Wittgensteins Bildtheorie von der Sprache. Wittgenstein geht nämlich „von der ikonischen Übereinstimmung der Sprache und [...] vor allem des Satzes mit der Wirklichkeit aus“. ${ }^{8}$ Sprache bildet somit die Welt ab, obwohl es sich dabei „nicht um die Abbildung von Gegenständen, sondern um die Abbildung von Relationen zwischen den Gegenständen" handelt. Gerade das macht Hermanns Aufgabe aus: Die Abbildung der von ihm postulierten Eins-zu-EinsRelation, die - in seiner Vorstellung - auf einer vollkommenen physischen Ähnlichkeit beruht. Worin besteht aber die Kernschwierigkeit, die das von Hermann erstrebte Vorhaben unmöglich macht? Ist die literarische Sprache wirklich nicht dazu fähig, ein überzeugendes bildhaftes Menschenporträt zu skizzieren? Keinesfalls. Das Problem liegt eher darin, dass die angesprochene Ähnlichkeit völlig imaginär ist und einzig in Hermanns Bewusstsein bzw. in seiner Phantasie existiert. Es ist also keine ,objektive Wirklichkeit', die er widerzuspiegeln sucht, sondern sein privates Hirngespinst; kein realer Gegenstand bzw. Sachverhalt, sondern ein subjektives Gefühl bzw. Gedankenphänomen. Die apodiktische Eindeutigkeit der Malerei würde dabei nicht helfen. Hätte Hermann tatsächlich Farben statt Worte benutzt, so wäre das Resultat für einen unbeteiligten Beobachter genauso irreführend wie die Realität. Die vermeintliche Affinität würde unbemerkt bleiben. Die Sprache aber - diejenige menschliche, literarische Sprache, deren Darstellungsmöglichkeiten Hermann dermaßen geringschätzt - kommt damit zurecht (wie auch mit vielen anderen komplexen Aufgaben ${ }^{10}$ ). Nur scheint sich der Sprechende dessen nicht bewusst zu sein. Die nächstentstehende Frage lautet: $W e r$, wenn nicht der Erzähler, weiß hier genau von der enormen Darstellungskapazität der Sprache und nutzt diese zwar spielerisch, aber mit voller Absicht aus? Die Antwort liegt nahe: Es ist der Erfinder der Erzählinstanz, also der Romanautor (in diesem konkreten Fall Vladimir Nabokov). Damit sind wir jedoch bei einem weiteren relevanten (und komplexen) Thema, welches im Folgenden kurz beleuchtet wird.

\section{Der Autor in statu nascendi}

Es ist wohl kein Zufall, dass die Titel der meisten bedeutenden Beiträge zur Theorie der Autorschaft - Michel Foucaults „Was ist ein Autor?"

$8 \quad$ Elisabeth Leiss, Sprachphilosophie, S. 138.

9 Ebd., S. 139.

10 James Wood macht eine sehr treffende Bemerkung, der Film habe die Montage-Technik vom Roman übernommen - und nicht umgekehrt, wie es oft behauptet wird. Vgl. James Wood, Die Kunst des Erzählens, Reinbek bei Hamburg 2016, S. 53-54.

11 Michel Foucault, Was ist ein Autor?, in: Fotis Jannidis, Gerhard Lauer, Mattias Martinez, Simone Winko (Hrsg.), Texte zur Theorie der Autorschaft, Stuttgart 2000, S. 198-233. 
Roman?“"12, Gérard Genettes „Implizierter Autor, implizierter Leser?" ${ }^{13}$, ja sogar Jean-Paul Sartres „Warum schreiben?" ${ }^{14}$ - ein Fragezeichen enthalten. Die heute als selbstverständlich wirkende Unterscheidung zwischen dem Autor eines fiktiven Werkes und dessen Erzählinstanz war zweifelsohne ein entscheidender und bedeutsamer (Fort)Schritt in der narratologischen Analyse literarischer Texte sowie in der gesamten Literaturwissenschaft. Macht man sich aber mit der beinahe unüberblickbaren Vielfalt von mittlerweile entstandenen Texten zur Dichotomie von Autor und Erzähler bekannt, so wird es offensichtlich, dass das Problem weder als gelöst noch als gar eindeutig festgelegt betrachtet werden kann.

Gérard Genette hat zweifelsohne Recht, wenn er behauptet, es sei im Erzählverfahren immer ein Ich präsent, sodass die Teilung in Ich- und Er-Erzählsituation streng genommen ziemlich eingeschränkt sei (denn irgendein Ich erhebt doch die Stimme und spricht). ${ }^{15}$ Gleichwohl ist der Er-Faktor bei der Erschaffung literarischer Texte nicht minder bedeutend als der Ich-Faktor. Für den Schreibenden ist nämlich jede von ihm erwählte/kreierte Erzählinstanz - egal ob sie in dritter oder auch in erster Person spricht - immer eine Er-Instanz. Es besteht also eine nicht zu unterschätzende Distanz zwischen dem (auto)biographischen und dem schreibenden Ich eines Autors. Der georgische Philosoph Merab Mamardaschwili (1930-1990), der sich unter anderem mit der Erkenntnistheorie beschäftigte, kommt in seinen Vorlesungen über Marcel Proust sowie im Essay „Аитературная критика как акт чтения” [Literaturkritik als Leseakt] zur ziemlich wagemutigen Schlussfolgerung, ein Roman sei etwas, was den sog. ,Autor ' erstmals ,produziert' (und nicht etwa umgekehrt). Der Autor ist nach Mamardaschwili keine selbständige, von seinem Text unabhängige Größe, sodass es keinerlei Message gibt, die der Schriftsteller den Lesern überliefern könnte. Im Gegenteil: Erst das niedergeschriebene Werk gibt seinem Autor die Möglichkeit, sich als Autor - im Sinne eines bewussten und erfüllten Individuums - zu betrachten. Die Ich-Existenz des Autors ist also kein natürliches Phänomen, daher geht sie dem Wort als Erfahrungsausdruck nicht voran, denn der Schriftsteller erkennt sich selbst erst durch den von ihm geschriebenen Text. ${ }^{16}$ Auch Proust glaubte, so Mamardaschwili, der Autor erfahre seine eigenen Gedanken und Gefühle nur indem er sie in Worte fasst und entsprechend strukturiere. ${ }^{17}$ Einen in etwa ähnlichen, obschon weniger erkenntnistheoretisch, sondern vielmehr dekonstruktiv gefärbten Standpunkt findet man bei Roland Barthes:

Der Autor [...] wird immer als die Vergangenheit seines eigenen Buches verstanden. Buch und Autor stellen sich in ein und dieselbe Reihe, unterschieden durch ein Vorher und Nachher. [...] Hingegen wird der moderne Schreiber [...] im selben Moment wie sein Text geboren. Er hat überhaupt keine Existenz, die seinem Schreiben voranginge oder es überstiege [...]. Es gibt nur die Zeit der Äußerung, und jeder Text ist immer hier und jetzt geschrieben. ${ }^{18}$

12 Wolfgang Kayser, Wer erzählt den Roman?, in: ebd., S. 127-142.

3 Gérard Genette, Implizierter Autor, implizierter Leser?, in: ebd., S. 233-251.

14 Jean-Paul Sartre, Warum schreiben, in: ebd., S. 106-127.

15 Vgl. Gérard Genette, Die Erzählung, München 1998, S. 257.

6 Vgl. Мераб Мамардашвили [Merab Mamardaschwili], $\Lambda$ итературная критика как акт чтения [Literaturnaja kritika kak akt tschtenija], in: Марсель Пруст, Заметки об искусстве и митературной критике [Zametki ob iskusstwe i literaturnoj kritike] (Notizen über Kunst und Literaturkritik), Moskau 2016, S. 14, 17. 17 Vgl. ebd.

18 Roland Barthes, Der Tod des Autors, in: Fotis Jannidis, Gerhard Lauer, Mattias Martinez, Simone Winko (Hrsg.), Texte zur Theorie der Autorschaft, Stuttgart 2000, S. 189. 
Diese ,erzählte Selbsterkenntnis' sowie die damit einhergehende sprachliche Autoreferentialität kann zweifelsohne als eines der Hauptmerkmale insbesondere der Literatur der Moderne betrachtet werden, obwohl ein eigenwilliger, dezent-revolutionärer Umgang mit Sprache und Form auch für die Texte aus früheren Epochen charakteristisch ist. Die übliche, normorientierte Sprachverwendung unterliegt in einem literarischen Werk einer Modifikation, welche, besonders in den klassischen Texten, leicht übersehen werden kann, denn im Gegensatz zu den Modernisten haben die klassischen Autoren ihre Kunstgriffe nicht so häufig und explizit offengestellt. Was sollte man aber in diesem Kontext unter der normorientierten Sprachverwendung verstehen? Wittgensteins Sprachphilosophie besprechend, resümiert Elisabeth Leiss einen bedeutenden Teil seiner Satzlehre folgendermaßen:

In analytischen Sätzen drücken nicht wir uns aus, so Wittgenstein, sondern es drückt sich die Natur der naturnotwendigen Zeichen aus. [...] Analytische Sätze explizieren semantische Merkmale, sind also Definitionen, wie z.B. in Junggesellen sind unverheiratet. Analytische Sätze enthalten also unser Wissen über die Welt, das intersubjektiv als wahr gilt und nur in Ausnahmefällen von der Sprachgemeinschaft neu formatiert wird. ${ }^{19}$

In einem literarischen Werk kann dieses Verhältnis zwischen dem Subjektiv-Intentionellen und dem Objektiv-Analytischen total umgedeutet werden, und zwar auf eine äußerst bezeichnende Art und Weise. Nehmen wir als Beispiel den klassischen Anfangssatz von Leo Tolstois „Anna Karenina“: „Alle glücklichen Familien sind einander ähnlich; jede unglückliche Familie ist auf $i$ ihre Weise unglücklich. ${ }^{“ 2}$ Ein zutiefst persönliches, ja umstrittenes Urteil wird hier in die Form einer allgemeinbekannten, intersubjektiven Wahrheit verkleidet. Der Satz wirkt demnach analytisch, denn er sollte vom Leser ähnlich empfunden werden wie eben Junggesellen sind unverheiratet. Was heißt hier aber „sollte“? Wer spielt mit dem Leser das eigenartige sprachliche Katz-und-Maus-Spiel? Diejenige Instanz, die sich durch diesen pseudoanalytischen Satz ausdrückt, ist nämlich nicht nur der Erzähler, sondern auch (oder gar vor allem) der Autor. Dabei darf man allerdings, wie bereits erwähnt wurde, nicht naiv-biographisch vorgehen und unter ,Autor' den Menschen Tolstoi, der von 1828 bis 1910 gelebt hat, verstehen. Vielmehr geht es um den Romanautor Tolstoi, dem Schöpfer von „Anna Karenina“, dessen rebellischer Wille bewirkt, dass man keinen gravierenden Unterschied sieht zwischen der obenzitierten Einleitungsthese und den nächstfolgenden Satz: „Im Hause der Oblonskiys herrschte allgemeine

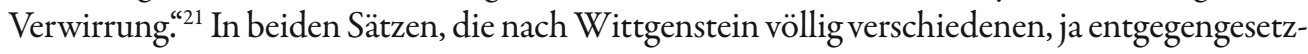
ten Sinnebenen angehören, wird bei Tolstoi „ein Wahrheitswert implizit und unhintergehbar

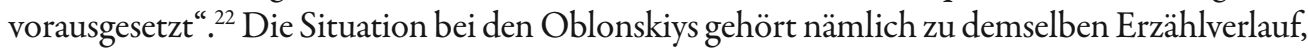
den der vorherige Satz (über glückliche und unglückliche Familien) in Bewegung setzte. Daher kann der Rezipient die am Anfang des Romans postulierte Idee genauso wenig negieren wie die ,Tatsache', dass im Hause Oblonskiy allgemeine Verwirrung herrschte.

19 Elisabeth Leiss, Sprachphilosophie, S. 142.

20 Lew Tolstoi, Anna Karenina. Erster Band, Kap. 2, URL: http://gutenberg.spiegel.de/buch/anna-kareninaerster-band-4043/2 [Zugriff am 05.05.2018].

21 Ebd.

22 Elisabeth Leiss, Sprachphilosophie, S. 141. 
Damit landen wir - überraschungsweise - wieder bei Kant. Leiss schreibt: „Kants Philosophie hat seine Zeitgenossen nicht wenig deprimiert. Attraktiv ist seine Philosophie dennoch

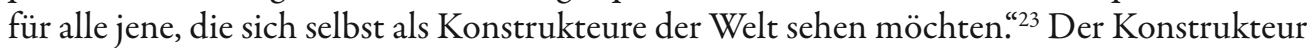
der Welt(en): Eine bessere Schriftsteller-Metapher könnte man wohl kaum erfinden.

\section{Die Dialektik des Unaussprechlichen}

Es wurde vorhin festgestellt, Literatur bilde eine besondere Art geistiger Tätigkeit, welche - kraft ihres extrem sprachfixierten Ausdrucksinstrumentariums - immer wieder mehrere autoreferentielle Züge aufweist sowie danach strebt, die Beschränktheit eigener Bezeichnungsmittel zu überwinden. Die Verzweiflung eines Dichters, der die stumpfe Nichtigkeit des Wortes ratlos beweint und der ärgerlichen Beengtheit literarischer Kunst die lebendig-expressive Ausdruckskraft der Musik gegenüberstellt, ist sowohl üblich als auch nachvollziehbar. Dies trifft besonders auf die Romantiker zu, welche Musik generell wesentlich höher als Literatur schätzten. So liest man z.B. in E.T.A. Hoffmanns Rezension der Fünften Symphonie Beethovens:

Wenn von der Musik als einer selbstständigen Kunst die Rede ist, sollte immer nur die InstrumentalMusik gemeint seyn, welche, jede Hülfe, jede Beymischung einer andern Kunst verschmähend, das eigenthümliche, nur in ihr zu erkennende Wesen der Kunst rein ausspricht. Sie ist die romantischte aller Künste, - fast möchte man sagen, allein rein romantisch. ${ }^{24}$

Bemerkenswerterweise hebt Hoffmann ausgerechnet Instrumentalmusik hervor und nicht etwa Zauberoper oder romantisches Kunstlied. Je unabhängiger ein Musikstück vom Text entfernt ist, desto musikalischer (und somit romantischer) ist sein Wesen. Geht es darum, das Unaussprechliche auszudrücken (und eben darin bestand ja eines der Hauptziele von Romantikern), so eignet sich Musik - laut gängiger Vorstellungen - viel besser dazu als Literatur. Man kann hier allerdings die Frage stellen: Was versteckt sich eigentlich hinter dem Begriff des Unaussprechlichen? Handelt es sich dabei um bestimmte jenseits der menschlichen Sprache existierende/empfundene Phänomene, die man mittels sprachlicher Zeichen kaum ausdrücken kann? Oder sind unter dem ,Unaussprechlichen' vielleicht gerade diejenigen Grenzen der Sprache zu verstehen, die nur mittels dieser Sprache erkannt und fixiert werden können? Anders formuliert: Verhält es sich nicht so, dass erst die Sprach- und Sprechfähigkeit dem Menschen überhaupt ermöglicht, das Konzept des Unaussprechlichen zu erarbeiten? So gesehen wird das Unaussprechliche zur bloßen Metapher ursprünglicher sprachlicher Begrenztheit, welche mit der erwähnten sehnsüchtigen Tendenz zur Transzendierung der Sprache unzertrennlich verbunden ist. Um Nietzsches Übermenschen-Appell nochmal aufzugreifen, diesmal aber in einer modifizierten Form: Die Sprache ist etwas, was überwunden werden muss.

23 Ebd., S. 118.

24 E.T.A. Hoffmann-Portal: E.T.A. Hoffmann als Musiker, URL: http://etahoffmann.staatsbibliothekberlin.de/leben-und-werk/musiker/ [Zugriff am 22.04.2018]. 
In der Literatur der Moderne wird dieses Streben zu einem vieldiskutierten Thema, besteht ja einer der konstitutiven Aspekte moderner Welt- und Kunstauffassung in jenem unwiederbringlich verlorenen Vertrauen auf die Erkenntnisfunktion der Sprache, welches in Hugo von Hofmannsthals exemplarischem „Brief des Lord Chandos an Francis Bacon“ (1902) ebenso prägnant und aussagekräftig wie poetisch manifestiert wird:

Mein Fall ist, in Kürze, dieser: Es ist mir völlig die Fähigkeit abhanden gekommen, über irgend etwas zusammenhängend zu denken oder zu sprechen. Zuerst wurde es mir allmählich unmöglich, ein höheres oder allgemeineres Thema zu besprechen und dabei jene Worte in den Mund zu nehmen, deren sich doch alle Menschen ohne Bedenken geläufig zu bedienen pflegen. Ich empfand ein unerklärliches Unbehagen, die Worte ,Geist', ,Seele‘ oder ,Körper' nur auszusprechen. ${ }^{25}$

Was in Hofmannsthals Erzählung entscheidend bezweifelt wird, ist gerade diejenige für die Spätrealisten (z.B. für Heinrich Böll) charakteristische Erschließung der Wahrheit aus der Sprache, welche fruchtbare Kommunikation, Freiheit und Solidarität ermöglicht. Daher fühlt sich Lord Chandos sowohl unfrei als auch von seiner nächsten Umgebung total abgekapselt und zu keinerlei Kommunikation fähig. Sein wachsender Zweifel an der Sprache führt ihn konsequenterweise zum radikalen Beschluss, auf jegliche sprachliche Kontakte zu verzichten. Kurz: Das sprachliche System wird nicht mehr als „Kommunikationsstruktur, die zwischen Individuum und Wirklichkeit vermittelt" ${ }^{26}$ betrachtet, sondern vielmehr als Störfaktor, der jegliche Welt-Mensch-Beziehung verdunkelt bzw. verhindert.

1954 erscheint mit "Stiller“ - dem Durchbruchsroman von Max Frisch - ein weiterer, den modernistischen Sprachskeptizismus neu reflektierender Text. Wieder steht das Problem der kommunikativen Funktion der Sprache im Zentrum der Überlegungen des an Identitätsstörungen leidenden Protagonisten. Die Relationen zwischen dem Schreibenden und der Sprache werden im Buch folgendermaßen geschildert:

Schreiben ist nicht Kommunikation mit Lesern, auch nicht Kommunikation mit sich selbst, sondern Kommunikation mit dem Unaussprechlichen. Je genauer man sich auszusprechen vermöchte, um so reiner erschiene das Unaussprechliche, das heißt die Wirklichkeit, die den Schreiber bedrängt und bewegt. Wir haben die Sprache, um stumm zu werden. ${ }^{27}$

Die wichtigste Frage, die dabei gestellt werden soll, betrifft die Möglichkeit bzw. die Bedingungen einer solchen Kommunikation. Der letzte Satz des angeführten Zitats bedeutet eigentlich nichts Anderes als dass die Sprache dem Menschen (wenigstens dem Schriftsteller) dazu gegeben wurde, um sie zu bekämpfen (im Sinne einer befreienden Transzendierung). Wie kommt es aber dazu, dass der Schreibende mit dem Unaussprechlichen mittels der Sprache kommuniziert? Um diese Dichotomie etwas besser zu verstehen, wäre es nicht unangebracht, sich einer gewissen Analogie aus der Philosophie des Buddhismus zu bedienen. Im Zentrum buddhistischer Mensch- und Lebenslehre stehen nämlich zwei Begriffe - Nirwana und

25 Hugo von Hofmannsthal, Der Brief des Lord Chandos und andere Schriften, Köln 2015, S. 37.

26 Klaus Torsy, Unser alltäglicher Wahnsinn. Zum Begriff der Kommunikation bei Dieter Wellershoff, Marburg 1999, S. 29.

27 Max Frisch, Stiller, Frankfurt a.M.1973, S. 330-331. 
Samsara - deren Relationen auf einer ebenso komplexen wie spannenden Dialektik basieren. Während das Nirwana als Zustand absoluter, vollendeter Ruhe (eine Art des erfüllten Nichtseins) gedeutet werden kann, so steht das Samsara für den endlosen Kreislauf von Geburt, Tod und Wiedergeburt. Ein durchschnittlicher, ,normaler ${ }^{\top}$ Mensch lebt in der Samsara-Wirklichkeit. Das Nirwana-Reich ist hingegen nur für denjenigen Erleuchteten zugänglich, dem es gelang, das Buddha-Bewusstsein in sich zu erwecken. Wie beziehen sich nun jene zwei Wirklichkeitsebenen zueinander? Einer der bedeutendsten russischen Denker des 20. Jahrhunderts und weltbekannter Buddhologe Alexander Piatigorsky erklärt dies so, dass die Welt des Samsara die Welt des Nirwana niemals, unter keinen Umständen erkennen kann, weil die Letztere unerkennbar ist (jedenfalls in den Samsara-Kategorien). Doch die Nirwana-Welt kann die Samsara-Welt durchaus erkennen. Das Nirwana erkennt somit nicht nur sich selbst, sondern auch das Samsara. ${ }^{28}$ Alles, was der wahren, letztendlichen Erkenntnis unterliegt, kann also einzig aus der NirwanaPerspektive erkannt werden, denn der Samsara-Perspektive bleibt sogar die eigentliche Selbstreflexion fremd (andernfalls würde das Samsara seine eigene illusorische Natur erkennen, was logischerweise unmöglich ist). Ähnlich verhält es sich mit der Sprache, dem Sprachverwender und dem Unaussprechlichen. Die Sprache kann - so der Ich-Erzähler von „Stiller“ - nur aus der Perspektive des Unaussprechlichen tatsächlich erkannt werden. Das Wort ,Kommunikation' wird dabei zwar benutzt, aber gleichzeitig scheint klar zu sein, dass diese sehr spezifische Art von Kommunizieren mit dem üblichen Sender-Empfänger-Dualismus wenig zu tun hat und vielmehr auf einer vollständigen Subjekt-Objekt-Verschmelzung beruht. Im Idealfall eines so verstandenen künstlerischen Meisterwerkes drückt die Sprache das Unaussprechliche nicht aus (dies wäre semantisch wie ontologisch fragwürdig), sondern wird eins damit; das Sagen löst sich im Unsagbaren auf. Wie ist es aber möglich und welche Mittel gibt es, um dies zu verwirklichen?

Eine überraschende Antwort gibt darauf der japanische Philosoph und Religionswissenschaftler Toshihiko Izutsu in seinem Buch „Philosophie des Zen-Buddhismus“, dessen drittes Kapitel vor allem sprachphilosophischen Problemen gewidmet ist. Dem Begriff der ,Artikulation' (im Sinne sprachlicher Markierung von Gegenständen, Gefühlen und Gedanken) ${ }^{29}$

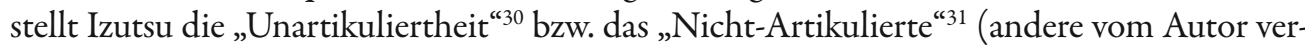
wendete Bezeichnungen sind „Nicht-Artikulation“, „Nicht-Differenzierung ${ }^{\text {“32 }}$ usw.) gegenüber, d.h. ein Verfahren, dank dem die ,endgültige Wirklichkeit ${ }^{“ 33}$ zum Ausdruck kommen kann. Was unter dieser endgültigen Wirklichkeit verstanden wird, beschreibt Izutsu als ein „metaphysisches Feld [...], das jenseits der Entzweiung in Subjekt und Objekt aktualisiert wird “34. Wie es in der Zen-Praxis oft der Fall ist, wird dies am Beispiel eines klassischen Kōans (eine kurze Sentenz oder Anekdote aus dem Leben und Lernen eines alten buddhistischen Meisters) erklärt:

28 Curiosity of intelligence: Александр Пятигорский. Аекции по фимософии будАизма. Конспект [Aleksandr Piatigorski, Lekcii po filosofii buddisma. Konspekt], URL: http://curiosityofintelligence.blogspot. com/2014/05/blog-post.html [Zugriff am 03.05.2018]..

${ }_{29}$ Toshihiko Izutsu, Philosophie des Zen-Buddhismus, Reinbek bei Hamburg 1986, S. 85.

30 Ebd., S. 95.

31 Ebd., S. 88.

32 Ebd., S. 87.

33 Ebd., S. 86.

34 Ebd. 
Ein Mönch fragte einst Meister Fūketsu: ,Das Sprechen verdirbt die Transzendenz (der Wirklichkeit), während das Schweigen die Manifestation verdirbt. Wie könnte man das Sprechen mit dem Schweigen vereinen, ohne die Wirklichkeit zu verderben?‘

Der Meister antwortete: ,Ich erinnere mich immer an die Frühlingslandschaft, die ich einst in Konan sah. Die Rebhühner riefen inmitten duftender Blumen in voller Blüte ${ }^{35}$.

Izutsu unterzieht diese Fabel einer exemplarischen Auslegung. Die Frage des jungen Mönchs betrachtet er als eines der zentralen Problem der Sprachphilosophie, nämlich: Ob die menschliche Sprache bzw. das menschliche Schweigen der „Wirklichkeit in ihrer ursprünglichen Ganzheit “36 jeweils gerecht werden können? Der beinahe verzweifelte Mönch sieht dies eher negativ. Er geht ja völlig berechtigt davon aus, dass der artikulierten Sprache stets eine differenzierend-begrenzende Funktion innewohnt, wogegen die Nicht-Artikulation über keine konkrete semantische Aufladung verfügt. Um es mit Izutsu zu formulieren:

Worte artikulieren Wirklichkeit in streng festgelegte Wesen (Einheiten). Auf der Ebene der linguistischen Darstellung kann es daher niemals eine freie Verbindung zwischen dem Vogel und der Blume geben. [...] Enthält man sich aber der Sprache, so geraten wir in eine andere Falle. Das Nicht-Artikulierte würde als reines ,Nichts' im ,negativen' Sinne des Wortes dastehen, was gerade das Gegenteil wäre von dem, was Zen behauptet. Denn für Zen kann das, was wir vorübergehend als das ,Nicht-Artikulierte' artikuliert haben, niemals getrennt von der unendlichen Vielfalt der Formen seiner Artikulation bestehen. ${ }^{37}$

Die philosophische Desorientierung des fragenden Mönches ist, so Izutsu, völlig verständlich und zeugt von seinem tiefen Verständnis der Zen-Lehre, denn er weiß ja, dass es sich bei dem Nicht-Artikulierten um kein bloßes ,Nichts' handeln kann. Also will er wissen, wie man die Artikulation mit der Unartikuliertheit vereinbaren könnte, ohne die Wirklichkeit zu verfehlen. Die Antwort des alten Meisters hat auf den ersten Blick mit der Frage seines Schülers nichts zu tun. Als wenn er die Worte des Mönches geradezu ignoriert hätte, entsinnt sich Fūketsu einer schönen Frühlingslandschaft, die er vor einiger Zeit an einem anderen Ort bewundern durfte. Gerade dieser Satz enthält aber, nach Izutsu, die exakteste Antwort, die auf die gestellte Frage gegeben werden konnte. Statt zu erklären, wie man Sprache und Schweigen bzw. Artikulation und Nicht-Artikulation miteinander verbinden kann, zeigt der Meister die Möglichkeit derartiger Synthese durch die von ihm produzierte Aussage, die im Gesamtkontext des Dialogs freilich etwas paradox klingt. Näher betrachtet, erweist sie sich jedoch als völlig legitim. Was ist es nun für eine Art und Weise, die Wirklichkeit zugleich artikuliert und nicht-artikuliert sprachlich darzustellen? Izutsu schreibt dazu Folgendes:

Interessanterweise antwortet Meister Fūketsu auf diese intellektuelle Frage mit der Darstellung der artikulierten und nicht artikulierten Wirklichkeit, indem er eine spezielle Funktion der menschlichen Sprache benutzt: Er gebraucht die Sprache dichterisch. ${ }^{38}$

35 Ebd., S. 90-91.

36 Ebd., S. 92.

37 Ebd., S. 91

38 Ebd., S. 92 
So gesehen gibt der alte Meister keine theoretische, sondern eine praktische Antwort auf die vermeintlich unlösbare Frage. Seine Erinnerungen an die einmal erlebte Frühlingslandschaft besitzen hier keinen informativen Wert. Der von ihm ausgesprochene Satz dient eben derjenigen Transzendierung bzw. Überwindung der Sprache, denn „die Artikulation verliert ihre funktionelle Grundlage ${ }^{\text {"39; }}$; die Sprache wirkt weder informativ noch kommunikativ, sondern dichterisch bzw. literarisch. In dieser Hinsicht ist die folgende Kernschlussfolgerung besonders wichtig, denn hierin werden eindeutige Parallelen zum Verhältnis der Sprache und des Unaussprechlichen sichtbar:

daß das Nicht-Artikulierte unter dem Aspekt der absoluten Unartikuliertheit mit keinen seiner artikulierten Formen, sei es auch dem sogenannten Absoluten, verwechselt werden darf. Viel wichtiger ist jedoch die dadurch unmittelbar implizierte Aussage, daß das Nicht-Artikulierte, gerade wegen seines wesentlichen Losgelöstseins von jeglicher artikulierter Form, alle artikulierten Formen annehmen kann. ${ }^{40}$

Versuchen wir nun, mit diesem Zitat eine ähnliche Operation zu wagen wie diejenige, die uns bei der Auseinandersetzung mit Kants Vernunftdialektik erlaubte, die sprachliche Dichotomie in einem anderen Licht zu betrachten. Ersetzen wir also ,Artikulation' durch ,Sprache', das ,Nicht-Artikulierte ' hingegen durch das ,Unaussprechliche'. Dann hieße die neue, unmittelbar auf Sprache und Literatur bezogene Pointe nämlich:

Das Unaussprechliche kann, gerade wegen seines wesentlichen Losgelöstseins von jeglicher sprachlicher Form, alle sprachlichen Formen annehmen.

\section{Fazit}

Wie oben bereits festgestellt wurde, tendiert die Sprache - ihrer ontologischen Natur nach - stets zur Selbstüberwindung und -transzendierung. Um diese möglichst erfolgreich zu vollziehen, muss sie sich aber dem sog. ,Unaussprechlichen' zuwenden, um schließlich in der Dichtung den Höhepunkt derartiger Manifestation zu finden. Den Dichtungsbegriff kann man dabei entweder wie Toshiko Izutsu im engen Sinn von Lyrik verstehen („Die artikulierende Funktion der dichtenden Sprache ist [...] völlig verschieden [...] von der artikulierenden Funktion der Prosa " "' , behauptet der japanische Philosoph) oder aber im weiteren Sinn von sämtlichen Sprachgebrauchsformen, welche einen hohen autoreferentiellen Grad aufweisen. Diese zweite Herangehensweise scheint angebrachter zu sein, bedenkt man z.B. die „progressive Universalpoesie “42 deutscher Romantiker, die ja die Barriere zwischen Poesie und Prosa aufzuheben suchten, oder Hugo von Hofmannsthals „Brief“. Nicht zufällig behauptete Nabokov, jedes große Literaturwerk sei in erster Linie ein Sprach- und nicht etwa ein Ideenphänomen ${ }^{43}$. Was die Literatur- oder

39 Ebd., S. 79.

40 Ebd., S. 95.

$41 \quad$ Ebd., S. 92.

42 Friedrich Schlegel, Progressive Universalpoesie, in: Hans-Jürgen Schmitt (Hrsg.), Die deutsche Literatur in Text und Darstellung. Romantik I, Stuttgart 2003, S. 22.

43 Vgl. ВАадимир Набоков (Vladimir Nabokov), Аекции по русской митературе [Lekcii po russkoj literature] (Vorlesungen über die russische Literatur), Moskau 2012, S. 111. 
Sprachwissenschaft betrifft, so sind sie allein wegen des Gegenstands ihrer Untersuchungen der autoreferentiellen bzw. metasprachlichen Vorgehensweise schicksalsmäßig ausgeliefert. Aber auch für andere sprachorientierte Disziplinen gilt eigentlich dieselbe ungeschriebene Regel. So stellt Vladimir Bibichin, einer der „einflussreichsten russischen Philosophen des letzten Jahrhunderts“ ${ }^{44}$ (in Deutschland vor allem als Heidegger-Übersetzer bekannt), am Ende seines Essays „Die Sprache der Philosophie“ fest, die Titelformulierung sei tautologisch, denn „Philosophie ist Sprache“45. Man durfte daher daran zweifeln, ob der Sprechende bzw. der Autor als ,Sprachbenutzer' (oder ,Sprachverwender') bezeichnet werden kann, denn die Frage, wer bzw. was hier von wem bzw. wovon benutzt wird - die Sprache von dem Menschen oder vielleicht andersherum - bleibt paradoxerweise offen.

\section{Literatur}

Barthes, Roland, Der Tod des Autors, in: Fotis Jannidis, Gerhard Lauer, Mattias Martinez, Simone Winko (Hrsg.), Texte zur Theorie der Autorschaft, Stuttgart 2000, S. 185-193.

[Bibichin, Wladimir] Бибихин, ВАадимир, Мир. Язык фикософии [Mir. Jazyk filosofii], SanktPetersburg 2015.

Curiosity, of intelligence: А^ександр Пятигорский. Аекции по философии буддизма. Конспект [Aleksandr Piatigorski, Lekcii po filosofii buddisma. Konspekt], URL: http://curiosityofintelligence.blogspot.com/2014/05/blog-post.html [Zugriff am 03.05.2018].

Foucault, Michel, Was ist ein Autor?, in: Fotis Jannidis, Gerhard Lauer, Mattias Martinez, Simone Winko (Hrsg.), Texte zur Theorie der Autorschaft, Stuttgart 2000, S. 198-233.

Frisch, Max, Stiller, Frankfurt a.M. 1973.

Genette, Gérard, Die Erzählung, München 1998.

Genette, Gérard, Implizierter Autor, implizierter Leser?, in: Fotis Jannidis, Gerhard Lauer, Mattias Martinez, Simone Winko (Hrsg.), Texte zur Theorie der Autorschaft, Stuttgart 2000, S. 233-251. von Hofmannsthal, Hugo, Der Brief des Lord Chandos und andere Schriften, Köln 2015.

Hoffmann-Portal E.T.A. : E.T.A. Hoffmann als Musiker, URL: http://etahoffmann.staatsbibliothekberlin.de/leben-und-werk/musiker/ [Zugriff am 22.04.2018].

Izutsu, Toshihiko, Philosophie des Zen-Buddhismus, Reinbek bei Hamburg 1986.

Kant, Immanuel, Kritik der reinen Vernunft, Köln 2011.

Kayser, Wolfgang, Wer erzählt den Roman?, in: Fotis Jannidis, Gerhard Lauer, Mattias Martinez, Simone Winko (Hrsg.), Texte zur Theorie der Autorschaft, Stuttgart 2000, S. 127-142.

Leiss, Elisabeth, Sprachphilosophie, Berlin 2009.

Lew, Tolstoi, Anna Karenina. Erster Band, Kap. 2, URL: http://gutenberg.spiegel.de/buch/annakarenina-erster-band-4043/2 [Zugriff am 05.05.2018].

[Mamardaschwili, Merab] Мамардашвики Мераб, Аитературная критика как акт чтения [Literaturnaja kritika kak akt tschtenija], in: Марсель Пруст (Marsel Prust), Заметки об искусстве и митературной критике [Zametki ob iskusstwe i literaturnoj kritike], Moskau 2016.

44 URL: https://www.matthes-seitz-berlin.de/buch/der-andere-anfang.html, Zugriff am 04.05.2018.

45 Vgl. [Wladimir Bibichin] ВАадимир Бибихин, Мир. Язык фимософии [Mir. Jazyk filosofii (Die Welt. Die Sprache der Philosophie)], Sankt-Petersburg 2015, S. 444: „Заглавие ,язык философии', как уже говоримось, тавтология. Философия и есть язык“" [ins Deutsche übersetzt von A.K.]. 
Nabokov, Vladimir, Die Verzweiflung, Reinbek bei Hamburg 2001.

[Nabokov, Vladimir] Набоков, ВАадимир, Аекции по русской Аитературе [Lekcii po russkoj literature], Moskau 2012.

[Piatigorski, Aleksandr] Пятигорский, Александр, Мышление и наблюдение. Четыре мекции по обсервационной фимософии [Myschlenie i nabljudenie. Tschetyre lekcii po obserwacionnoj filosofii], Moskau 2016.

Sartre, Jean-Paul, Warum schreiben, in: Fotis Jannidis, Gerhard Lauer, Mattias Martinez, Simone Winko (Hrsg.), Texte zur Theorie der Autorschaft, Stuttgart 2000, S. 106-127.

Schlegel, Friedrich, Progressive Universalpoesie, in: Hans-Jürgen Schmitt (Hrsg.), Die deutsche Literatur in Text und Darstellung. Romantik I, Stuttgart 2003, S. 22-25.

Stramiello, Tanja, Der Begriff des Übermenschen bei Friedrich Nietzsche, Hamburg 2014.

Tolstoi, Lew, Anna Karenina. Erster Band, Kap. 2, URL: http://gutenberg.spiegel.de/buch/annakarenina-erster-band-4043/2 [Zugriff am 05.05.2018].

Torsy, Klaus, Unser alltäglicher Wahnsinn. Zum Begriff der Kommunikation bei Dieter Wellershoff, Marburg 1999.

Wood, James, Die Kunst des Erzählens, Reinbek bei Hamburg 2016. 